\title{
Molecular Screening of Small Biopsy Samples Using Next-Generation Sequencing in Korean Patients with Advanced Non-small Cell Lung Cancer: Korean Lung Cancer Consortium (KLCC-13-01)
}

Bo Mi Ku* $\cdot$ Mi Hwa Heo ${ }^{1 *}$ Joo-Hang Kim${ }^{2} \cdot$ Byoung Chul Cho ${ }^{3}$ Eun Kyung $\mathrm{Cho}^{4}$. Young Joo Min ${ }^{5}$ Ki Hyeong Lee ${ }^{6} \cdot$ Jong-Mu Sun ${ }^{1}$ Se-Hoon Lee ${ }^{1}$. Jin Seok Ahn ${ }^{1}$ Keunchil Park ${ }^{1}$. Tae Jung Kim ${ }^{7}$ Ho Yun Lee · Hojoong Kim ${ }^{8}$ Kyung-Jong Lee ${ }^{8} \cdot$ Myung-Ju Ahn ${ }^{1}$

Samsung Biomedical Research Institute, Sungkyunkwan University School of Medicine, Seoul; ' ${ }^{2}$ ivision of Hematology-Oncology, Department of Medicine, Samsung Medical Center, Sungkyunkwan University School of

Medicine, Seoul; ' ${ }^{2} \mathrm{CHA}$ Bundang Medical Center, CHA University, Seongnam; ${ }^{3}$ Division of Medical Oncology, Yonsei Cancer Center, Seoul; ${ }^{4}$ Division of Hematology and Medical Oncology, Department of Internal Medicine, Gachon University Gil Medical Center Incheon; ${ }^{5}$ Division of Oncology, Department of Hematology and Oncology, Ulsan University Hospital, Ulsan; ${ }^{6}$ Division of Medical Oncology, Chungbuk National University Hospital, Chungbuk National University College of Medicine, Cheongju; ${ }^{7}$ Department of Radiology and Center for Imaging Science, Samsung Medical Center, Sungkyunkwan University School of Medicine, Seoul: ${ }^{8}$ Division of Pulmonary and Critical Care Medicine, Department of Medicine, Samsung Medical Center, Sungkyunkwan University

School of Medicine, Seoul, Korea

Received: December 28, 2017

Revised: February 26, 2018

Accepted: March 12, 2018

Corresponding Author

Kyung-Jong Lee, MD

Division of Pulmonary and Clinical Care Medicine,

Department of Medicine, Samsung Medical Center,

Sungkyunkwan University School of Medicine,

81 Irwon-ro, Gangnam-gu, Seoul 06351, Korea

Tel: +82-2-3410-0777

Fax: +82-2-3410-6956

E-mail: kj2011.lee@samsung.com

Myung-Ju Ahn, MD

Division of Hematology-Oncology, Department of

Medicine, Samsung Medical Center,

Sungkyunkwan University School of Medicine, 81

Imon-ro, Gangnam-gu, Seoul 06351, Korea

Tel: +82-2-3410-3438

Fax: +82-2-3410-1754

E-mail: silk.ahn@samsung.com

${ }^{*}$ Bo Mi Ku and Mi Hwa Heo contributed equally to this work.
Background: Non-small cell lung cancer (NSCLC) is a common type of cancer with poor prognosis. As individual cancers exhibit unique mutation patterns, identifying and characterizing gene mutations in NSCLC might help predict patient outcomes and guide treatment. The aim of this study was to evaluate the clinical adequacy of molecular testing using next-generation sequencing (NGS) for small biopsy samples and characterize the mutational landscape of Korean patients with advanced NSCLC. Methods: DNA was extracted from small biopsy samples of 162 patients with advanced NSCLC. Targeted NGS of genomic alterations was conducted using lon AmpliSeq Cancer Hotspot Panel v2. Results: The median age of patients was 64 years (range, 32 to 83 years) and the majority had stage IV NSCLC at the time of cancer diagnosis (90\%). Among the 162 patients, 161 patients (99.4\%) had novel or hotspot mutations (range, 1 to 21 mutated genes). Mutations were found in 41 genes. Three of the most frequently mutated genes were TP53 (151, 93.2\%), KDR (104, 64.2\%), and epidermal growth factor receptor (EGFR; 69, 42.6\%). We also observed coexistence of EGFR and other oncogene (such as KRAS, PIC3CA, PTEN, and STK11) mutations. Given that $69.6 \%$ (48/69) of EGFR mutant patients were treated with EGFR tyrosine kinase inhibitors, EGFR mutant status had higher prognostic ability in this study. Conclusions: These results suggest that targeted NGS using small biopsy samples is feasible and allows for the detection of both common and rare mutations in NSCLC.
Key Words: Carcinoma, non-small cell lung; Targeted next-generation sequencing; Small biopsy; Receptor, epidermal growth factor 
The majority of patients diagnosed with non-small cell lung cancer (NSCLC) present with advanced stage disease and have extremely poor prognosis. ${ }^{1}$ Recent advances in the understanding of lung cancer biology and improvements in technology have allowed molecular classification of NSCLC., ${ }^{2,3}$ Classifying NSCLC into distinct actionable subtypes with mutually exclusive driver oncogenes has led to the development of targeted therapy. ${ }^{3}$ Better survival has been observed after treatment with epidermal growth factor receptor (EGFR) or anaplastic lymphoma kinase (ALK) tyrosine kinase inhibitors (TKIs) in patients harboring the appropriate activating mutation or translocation, compared with standard chemotherapy., ${ }^{3,4}$

Although several genes including EGFR and $A L K$ have been identified as potential oncogenic drivers and targets for therapy, a large fraction of NSCLC patients do not have mutations in these commonly mutated genes. Thus, there are needs to identify additional driver oncogenes and targets for treatment. In addition, many NSCLC patients also harbor other co-existing molecular alterations that might influence the efficacy of a targeted therapy, leading to primary or secondary resistance. It is important to investigate these concurrent genetic alterations to reveal clinically significant predictive and prognostic markers. However, several challenges remain in the implementation of multiple molecular tests to find therapeutic or prognostic markers. First, most NSCLC biopsy samples are not amenable to multiple molecular tests due to the small amounts of tissues obtained by bronchoscopy or core biopsy. In addition, conventional molecular tests such as Sanger sequencing and polymerase chain reaction (PCR) are insensitive to alterations occurring at allele frequencies lower than $20 \%$. Finally, multiple and separate tests result in higher costs and longer turn-around time. Thus, a more comprehensive, sensitive, and time/cost-effective multiplex test is necessary to optimize the application of targeted therapy. ${ }^{5,6}$ Consequently, incorporation of molecular screening using nextgeneration sequencing (NGS) in the pathologic evaluation of NSCLC is now considered the standard in clinical practice. ${ }^{7.8}$

The rapid development of NGS technologies has enabled a new paradigm in precision medicine for oncology. It is now possible to identify oncogenic alterations that would have been previously undiscovered by conventional tests such as sequencing. For the routine clinical molecular diagnostic testing in NSCLC, NGS need to meet some criteria; NGS platform should be able to detect targetable driver mutations from limited amounts of input DNA from small biopsy or cytology samples, the turnaround time should be short, and the cost should be low. Unlike whole-genome sequencing or whole-exome sequencing, targeted
NGS including selected genes that show frequent alterations in cancer can reduce the amount of tissue, time, and cost required for testing. ${ }^{9-11}$

To validate the accuracy and feasibility of targeted NGS, we used Ion Ampliseq Cancer Hotspot Panel v2 to identify the variety of tumor-associated mutations in formalin-fixed paraffin-embedded (FFPE) or fresh frozen (FF) specimens from 162 advanced NSCLC patients in Korea. In this study, we analyzed multiple somatic mutations found in our advanced NSCLC cohort in order to detect known actionable mutations and discover potential therapeutic targets and prognostic biomarkers for NSCLC.

\section{MATERIALS AND METHODS}

\section{Patients and tumor samples}

We analyzed 162 FFPE or frozen tumor tissue specimens from advanced NSCLC patients between January 2014 and December 2015 at Samsung Medical Center (SMC). All samples were collected before any treatments were initiated. Procedures used for tumor tissue sampling varied, including videoassisted thoracoscopic surgery, core-needle biopsy, bronchoscopy, and endobronchial ultrasonography. Clinical data were obtained retrospectively from electronic medical records. The clinical variables assessed were sex, age at diagnosis, smoking history, tumor subtype, cancer stage, EGFR mutation, $A L K$ rearrangement, chemotherapy regimen, TKIs, and tumor response. Separately, EGFR mutation status was tested by real-time PCR using the peptide nucleic acid (PNA)-clamping EGFR Mutation Detection Kit (Panagene, Inc., Daejeon, Korea). Real-time PCR was performed using a CFX96 (Bio-Rad, Hercules, CA, USA) and all reagents were included in the kit. PCR cycling and mutation detection were done as previously described. ${ }^{12} A L K$ rearrangement status was tested by immunohistochemistry and confirmed by fluorescence in situ hybridization (FISH). All procedures involving tumor specimens were reviewed and approved by the Institutional Review Board (IRB) of SMC and all data were fully anonymized (SMC 2013-08-113-020). Written informed consent was provided by all patients.

\section{DNA extraction}

All tissue sections were reviewed by pathologists, and only those with tumor content more than $10 \%$ were included in the study. Genomic DNA was extracted from FFPE samples using the QIAamp DNA FFPE Tissue Kit (Qiagen, Hilden, Germany) or FF tumor specimens using QIAamp DNA mini kit. Purified DNA was quantitated by NanoDrop (Invitrogen Life Technolo- 
gies, Carlsbad, CA, USA) and Qubit Fluorometer (Invitrogen Life Technologies).

\section{Next-generation sequencing and data analysis}

The Ion Torrent Ion AmpliSeq Cancer Hotspot Panel v2 (Life Technologies) was used. This panel detects hotspot regions, including 2,800 COSMIC mutations of 50 oncogenes and tumor suppressor genes. A total of 162 cases of NSCLC specimens were subjected to NGS on the AmpliSeq platform. Sequencings were done according to previously described methods. ${ }^{13}$ Variants calls were further processed to reduce potential false-positives. Coverage $(>500 \times)$ was considered as filtering criteria and the minimal variant allele frequency was $2 \%$ for confirming variants as real. After filtering using these criteria, variants causing amino acid change and frameshift were finally used for statistical analysis.

\section{Statistical analysis}

Clinical and radiological response to treatment was assessed according to Response Evaluation Criteria In Solid Tumor ver. 1.1. Kaplan-Meier estimates were used for the analysis of all time-to-event variables. Progression-free survival (PFS) was calculated from the date of chemotherapy to the date of disease progression or death from any cause or the date of last followup. The overall survival (OS) was measured from the date of chemotherapy to the date of death from any cause and was censored at the date of the last follow-up visit. Variables with $\mathrm{p}<$ .05 were considered significant. All statistical analyses were performed using PASW Statistics ver. 23.0 (SPSS Inc., Chicago, IL, USA).

\section{RESULTS}

\section{Patient characteristics}

The clinical characteristics of advanced NSCLC patients included in the present study are summarized in Table 1 . The median age was 64 years (range, 32 to 83 years) and gender proportions were roughly equal (male $57 \%$ vs female $43 \%$ ). Seventy-nine patients (59\%) were smokers or former smokers, 83 patients (51\%) were never smokers. The NSCLC subtype distribution was as follows: adenocarcinoma (139/162, 85.8\%), squamous cell carcinoma $(17 / 162,10.5 \%)$, adenosquamous cell carcinoma $(1 / 162,0.6 \%)$, and other $(5 / 162,3.1 \%)$. The majority of patients had stage IV NSCLC at the time of cancer diagnosis (145/162; 90\%). In stage IV NSCLC patients, the median PFS was 6.2 months $(95 \% \mathrm{CI}, 4.2$ to 8.1$)$ and OS was 19.6 months $(95 \%$ CI, 15.4 to 23.7). EGFR mutation test was done in 145 patients,
Table 1. The baseline characteristics of patients

\begin{tabular}{|c|c|}
\hline Characteristic & No. $(\%)(n=162)$ \\
\hline \multicolumn{2}{|l|}{ Age (yr) } \\
\hline Median & 64 \\
\hline Range & $32-83$ \\
\hline \multicolumn{2}{|l|}{ Sex } \\
\hline Male & $92(57)$ \\
\hline Female & $70(43)$ \\
\hline \multicolumn{2}{|l|}{ Smoking history } \\
\hline Never-smoker & $83(51)$ \\
\hline Current & $33(20)$ \\
\hline Ex-smoker & $46(28)$ \\
\hline \multicolumn{2}{|l|}{ Histology } \\
\hline Adenocarcinoma & $139(86)$ \\
\hline Squamous cell carcinoma & $17(10)$ \\
\hline Adenosquamous & $1(1)$ \\
\hline NSCLC, other & $5(3)$ \\
\hline \multicolumn{2}{|l|}{ Clinical stage } \\
\hline $\mid-\|$ & $7(4)$ \\
\hline$\|I\| A$ & $5(3)$ \\
\hline$\| I \mid \mathrm{B}$ & $5(3)$ \\
\hline IV & $145(90)$ \\
\hline \multicolumn{2}{|l|}{ Brain metastasis } \\
\hline Present & $49(30)$ \\
\hline Absent & $113(70)$ \\
\hline \multicolumn{2}{|l|}{ Biopsy type } \\
\hline VATS & $45(28)$ \\
\hline CNB_lung & $23(14)$ \\
\hline CNB_others & $22(14)$ \\
\hline Bronchoscopy & $31(19)$ \\
\hline EBUS & $41(25)$ \\
\hline \multicolumn{2}{|l|}{ First treatment } \\
\hline Chemotherapy & $81(50)$ \\
\hline EGFR TKI & $51(32)$ \\
\hline ALK TKI & $3(2)$ \\
\hline No treatment & $27(16)$ \\
\hline
\end{tabular}

NSCLC, non-small cell lung carcinoma; VATS, video-assisted thoracoscopic surgery; CNB, core-needle biopsy; EBUS, endobronchial ultrasonography; EGFR, epidermal growth factor receptor; TKI, tyrosine kinase inhibitor; ALK, anaplastic lymphoma kinase.

and the mutation was detected in 64 patients $(44.1 \%)$ by realtime PCR using PNA-clamping. Positive results for $A L K$ rearrangement by FISH were detected in 14 patients (8.6\%). In this cohort, 81 patients were treated with cytotoxic chemotherapy (81/162, 50.0\%), 51 with EGFR TKIs (51/162, 31.5\%), and three with ALK TKIs $(3 / 162,1.8 \%)$ as first-line therapy.

\section{Molecular profiling of advanced NSCLC}

We employed targeted NGS technology to evaluate somatic mutations occurring in advanced NSCLC, using the Ion Torrent Ion AmpliSeq Cancer Hotspot Panel. Among the detected mutations, only those annotated in the Catalogue of Somatic Muta- 
tions in Cancer (COSMIC) database were considered. Mutations were found in 41 genes and commonly detected in the following genes: TP53 (151, 93.2\%), KDR (104, 64.2\%), EGFR (69, $42.6 \%)$, APC $(51,31.5 \%)$, RB1 $(30,18.5 \%)$, SMAD4 (28, $17.3 \%), \operatorname{MET}(22,13.6 \%)$, STK11 (20, 12.3\%), RET (18, $11.1 \%), \operatorname{ALK}(17,10.5 \%)$, and $\operatorname{KRAS}(13,8.0 \%)$, as shown in Fig. 1. Only one patient had no mutation while $161(99.4 \%)$ patients possessed more than one mutation (range, 1 to 21; median, 4). The vast majority of identified mutations were single nucleotide variant (SNV) followed by deletion (Del) and insertion (Ins). In accordance with the frequency described in previous studies, ${ }^{1,3}$ EGFR mutations were found in $42.6 \%$ patients and most of them $(54 / 69,78.3 \%)$ were typical mutations (30 Del exon 19 and 24 L858R). One of these was a triple EGFR mutant (L858R/G873R/Q787L) and ten were double EGFR mutant (3 Del exon 19/G873R, 2 Del exon 19/A750P, 1 Del exon 19/ S752Y, 1 Del exon 19/K754N, 2 L858R/G873R, 1 L858R/ T790M). Besides TP53 and EGFR, the most frequently mutated gene was $K D R$, and $K D R$ mutations appeared in codon 472 (103 Q472H) and codon 875 (1 T875A). One patient with KDR Q472H had concurrent KDR S1148C. MET mutations were found in codon 375 (17 N375S) and codon 179 (5 A179T). Although one MET R970C in exon 14 was simultaneously found with $\mathrm{N} 375 \mathrm{~S}$, this has not been reported as cause of MET exon 14 skipping. ${ }^{14}$ STK11 mutations were found in codon 354 (19 F354L) and codon 176 (1 D176G). Two samples detecting STK11 F354L had other STK11 mutation in codon 281 (P281L). Seventeen ALK mutations were all in codon 1184 (G1184E). In concordance with the known frequency of KRAS mutations in Asian population $(5 \%-10 \%),{ }^{15}$ they were found in $8 \%$ of this cohort. Most KRAS mutations appeared in codon 12 (1 G12A, 4 G12C, 3 G12D, 1 G12R, and 2 G12V) with two mutations in codon 50 (T50P). PIK3CA mutations (E81K, R401Q, E542G, E545A, E545K, Q546K, and H1047R) were detected in seven samples and one patient had double PIK3CA mutants (E542G/E545A). PTEN mutations (K66E, R130X, Q171X, and P246L) were identified in four patients.

We also observed co-occurrence of some of the most frequently mutated and clinically significant genes. Five patients simultaneously had mutations in both EGFR and KRAS. EGFR mutations also harbored PIK3CA and PTEN mutations, which were detected in three and two patients, respectively. In addition, although STK11 mutations were most commonly seen in association with KRAS mutations, we found seven cases with co-occurrence of EGFR and STK11 mutations in this study.

\section{Comparison of mutational profiles obtained with the AmpliSeq assay}

Based on mutation results considering the location of mutation sites, EGFR mutations were consistently detected by tar-

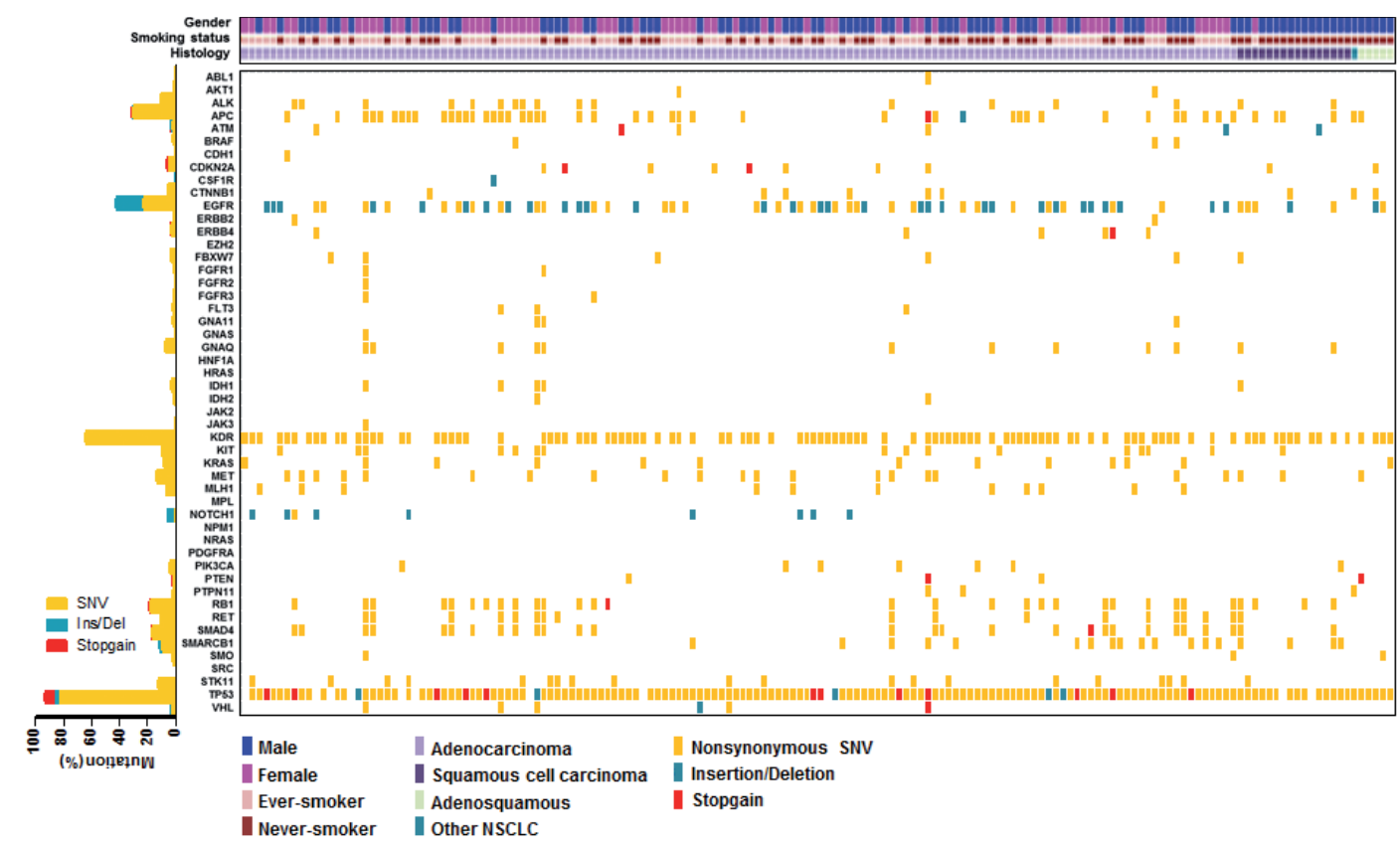

Fig. 1. Heatmap of mutations found in 162 non-small cell lung cancer samples. In the upper panel, the first row indicates sex, the second row smoking status, and the third row histology. A histogram shows the percentage of mutations in each gene (let). The horizontal axis presents the complete dataset of patients and the vertical axis illustrates mutated genes (right). 
geted NGS using AmpliSeq Cancer Panel and conventional PNA-clamping PCR (42.6\% vs 44.1\%). In 145 patients tested for $E G F R$ mutation, the comparison results of EGFR mutations detected by targeted NGS and conventional PNA-clamping PCR are summarized in Table 2. When comparing mutation detection of EGFR in FFPE and FF samples, a high concordance rate (92.4\%) was seen between NGS and PNA-clamping PCR. However, targeted NGS method identified additional EGFR mutations in 14 concordant cases and seven discordant cases that were not identified by PNA-clamping PCR. The most frequently found additional EGFR mutation was G873R which was found in 12 patients. We observed three discordant cases that showed positive results (all Del exon 19) in PNAclamping, but negative in NGS. Considering the high sensitivity of NGS, these results may be due to tumor heterogeneity.

Although most of the patients had a single biopsy, four patients had repeated biopsies and had double tumors tested. Except for one consistent case, the other three cases showed slightly different mutation profiles (Table 3). These differences may be due to tumor heterogeneity or tumor evolution.

\section{Impact of mutation status on survival}

We evaluated the relationships between $E G F R$ somatic mutations and survival. Activating EGFR mutations have been reported as prognostic factors in other studies. ${ }^{16,17}$ In this study cohort, $69.6 \%$ (48/69) of patients with EGFR mutations were treated with EGFR TKIs. The presence of EGFR mutations were definitive predictive markers of both PFS (hazard ratio [HR], 2.59; 95\% confidence interval [CI], 1.75 to 3.85) (Fig. 2A) and OS (HR, 2.00; 95\% CI, 1.30 to 3.09) (Fig. 2B). Median PFSs were 3.8 months for EGFR wild-type group and 14.6 months for EGFR mutant group. The median OS for EGFR

Table 2. Comparison of EGFR mutations detected by targeted NGS and PNA-clamping PCR

\begin{tabular}{|c|c|c|c|c|c|c|c|}
\hline \multirow{2}{*}{ Sample type } & \multirow{2}{*}{ No. of cases compared } & \multicolumn{2}{|c|}{ Concordant (NGS/PNA) } & \multicolumn{3}{|c|}{ Discordant (NGS/PNA) } & \multirow{2}{*}{ Concordance (\%) } \\
\hline & & $-1-$ & $+/+$ & $-/+$ & $+/-$ & $+/+$ & \\
\hline FFPE & 131 & 69 & 53 & 2 & 6 & 1 & 93.1 \\
\hline FF & 14 & 5 & 7 & 1 & 1 & 0 & 85.7 \\
\hline Total & 145 & 74 & 60 & 3 & 7 & 1 & 92.4 \\
\hline
\end{tabular}

EGFR, epidermal growth factor receptor; NGS, next-generation sequencing; PNA, peptide nucleic acid; PCR, polymerase chain reaction; FFPE, formalin-fixed paraffin-embedded; FF, fresh frozen.

Table 3. Mutations identified in four patients with repeat biopsy samples

\begin{tabular}{lll}
\hline Patient No. & \multicolumn{1}{c}{ Mutations identified in first sample } & \multicolumn{1}{c}{ Mutations identified in second sample } \\
\hline 1 & KDR Q472H, APC A1582P, MET N375S, TP53 R248W, TP53 P72R & KDR Q472H, APC A1582P, TP53 R248W, TP53 P72R \\
2 & ERBB4 T926M, KIT M541L, TP53 H179R, TP53 P72R & ERBB4 T926M, KIT M541L, FLT F590L, TP53 H179R, TP53 P72R \\
3 & PIK3CA E542K, PTPN11 G503V, TP53 E285K, TP53 P72R, TP53 & KIT M541L, KDR Q472H, TP53 P72R \\
P72A, SRC Q529X & CTNB1 D32A, KDR Q472H, EGFR ex19 del, EGFR A750P, CDKN2A \\
4 & CTNNB1 D32A, KDR Q472H, EGFR ex19 del, EGFR A750P, CDKN2A & $\begin{array}{c}\text { CTN6R, TP53 P72R } \\
\text { H66R, TP53 P72R }\end{array}$ \\
\hline
\end{tabular}
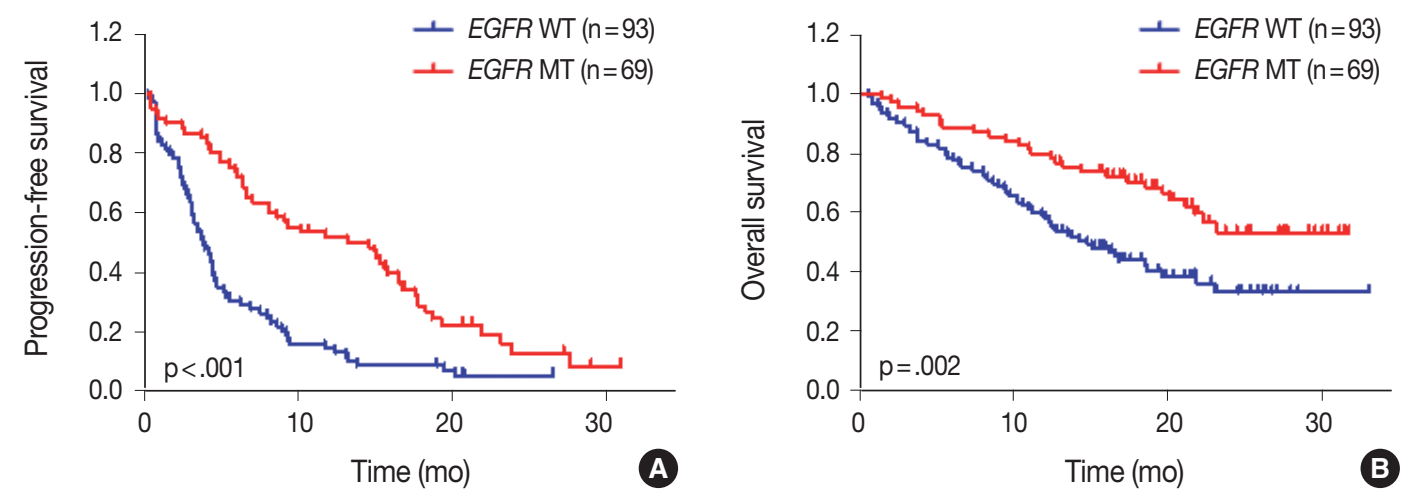

Fig. 2. Impact of epidermal growth factor receptor (EGFR) mutational status on survival. (A) Progression-free survival of patients with EGFR mutant (MT) compared with EGFR wild-type (WT) patients. (B) Overall survival of all patients according to EGFR status. p-values were obtained using the log-rank test. 

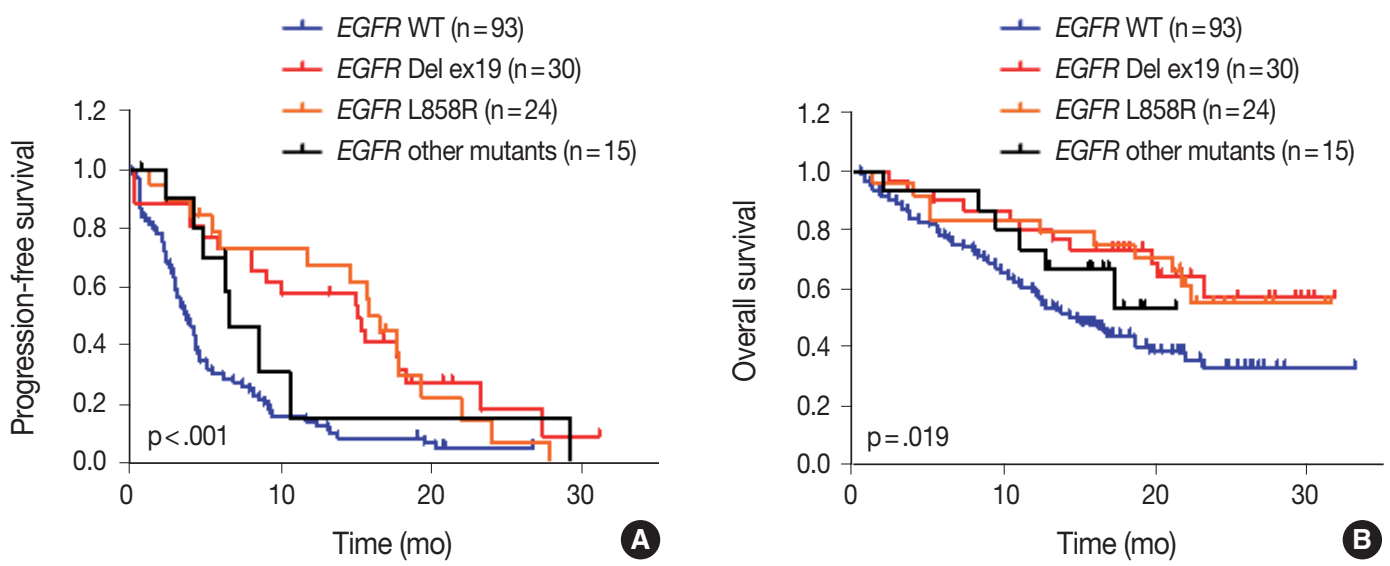

Fig. 3. Impact of different types of epidermal growth factor receptor (EGFR) mutations on survival. Progression-free survival (A) and overall survival (B) of patients with different types of EGFR mutations compared with EGFR wild type (WT) patients. p-values were obtained using the log-rank (Mantel-Cox) test.

wild-type group was 14.8 months, but the median OS for EGFR mutant group was not reached. When PFS was analyzed after grouping the patients according to the three types of EGFR mutations (Del ex19, L858R, and others), the median PFSs were different between activating mutations (15.1 months for Del ex19 and 17.7 months for L858R) and others (6.6 months) (Fig. 3A). However, the median OS was not reached in the three types of EGFR mutations (Fig. 3B).

\section{DISCUSSION}

Currently, molecular testing for $E G F R$ mutations and $A L K$ rearrangements is essential for targeted therapy in patients with NSCLC. However, the heterogeneous nature of NSCLC can lead to inaccurate molecular classification and therapeutic resistance. Other genetic alterations that have been found and have potential therapeutics include ROS1, RET, and NTRX gene rearrangement, MET exon 14 skipping, and MET amplification. ${ }^{4,18,19}$ However, there are no standard molecular diagnostic tests for these genetic alterations. In addition, an important limitation in current routine diagnosis is that the quantity of DNA extracted from small biopsy samples (FFPE or FF) is not adequate for multiple molecular tests in most cases. To cope with this limitation, comprehensive multiplex testing using NGS is necessary to improve the efficacy of targeted therapy for NSCLC patients. With advances in NGS technology, several target regions of interest can be sequenced concurrently and thereby improve the chances of identifying rare mutations. In this study, 162 Korean advanced NSCLC samples were assessed for mutations in oncogenes and tumor suppressor genes using an NGS platform (AmpliSeq Cancer Hotspot Panel). This targeted sequencing method shows high accuracy and requires only small quantities of sample (10 ng DNA), enabling researchers to sequence challenging small biopsy samples such as FFPE. Genetic alterations were confirmed in $99.4 \%$ of samples and 14 additional EGFR mutations (L707F, G719A, G719C, L747S, A750P, S752Y, K754N, S768I, V769L, V774M, T783A, S784P, Q787L, and G873R) were identified that were not detected with PNAclamping PCR. In addition, we found some of the most frequently altered and clinically significant genes such as KRAS, MET, STK11, PIK3CA, and PTEN mutations. Moreover, the higher sensitivity of NGS platform should increase the identification of concomitant mutations. These results suggest the feasibility and usefulness of targeted sequencing to identify low frequency mutations and detect additional mutations that are helpful to understand the clinical outcomes of the patients in each group.

For patients with EGFR-mutant NSCLC, EGFR TKIs are found to increase response rates and survival time. ${ }^{16,17}$ In concordance with these data, EGFR mutations were associated with significant improvements of PFS and OS compared to EGFR wild-type patients, because most patients were treated with EGFR TKIs. Despite these benefits of EGFR TKIs, not all patients respond to treatment and most EGFR-mutant NSCLC patients develop acquired resistance.

Tumor suppressor TP53 mutations are frequently detected in most human cancers. TP53 was also the most commonly altered gene in this study, and this result is consistent with those of previous studies. ${ }^{20,21}$ TP 53 was concurrently mutated with many other genes such as EGFR and KRAS in this study, perhaps due to the high frequency of TP53 mutations found in our samples. Whereas the frequency of TP53 mutation is well known, therapeutic options based on this alteration are scarce and controversial 
in patients with lung cancer. A previous study on advanced NSCLC found an association between TP53 mutation and shorter median OS, but another study, on the other hand, reported no association between TP53 mutation and survival. ${ }^{22,23}$

Our data also identified KDR Q472H polymorphism in 103 patients ( 31 homozygotes and 72 heterozygotes). KDR Q472H has been reported to increase tumor microvasculature and shown to mediate vascular endothelial growth factor receptor 2 phosphorylation in NSCLC. ${ }^{24}$ Furthermore, KDR Q472H had a higher proliferative and invasive capacity in melanoma. ${ }^{25}$ Although we did not find a significant correlation between KDR Q472H and survival in EGFR TKI- or chemotherapytreated NSCLC patients, the prognostic value of KDR Q472H should be different after treatment with vascular endothelial growth factor pathway inhibitors.

Although KRAS mutations are the most common oncogenic driver, there are some ethnic differences. The frequency of KRAS mutations in Asian is 5\%-15\%. In addition, KRAS mutations usually occur in $E G F R$ wild-type tumors. ${ }^{18,26}$ In this study, we detected 13 KRAS mutations (8\%) and five concurrent KRAS/ EGFR mutations (3.0\%) via NGS. In these five patients, three patients, who were clinically confirmed to have EGFR L858R mutations, received EGFR TKI (gefitinib) treatment with partial response or progression of the disease. Other two patients were treated with chemotherapy and showed 0.8 and 9.3 months of PFS. Although the prognostic effect of KRAS mutations was not clear due to small sample size, these results suggest that KRAS mutation test using NGS platform may help determine the appropriate therapy for NSCLC patients.

Recent studies have demonstrated that mutations in EGFRdownstream genes such as PIK3CA, PTEN, and STK11 are associated with de novo resistance to EGFR TKI. ${ }^{27}$ Furthermore, PIK3CA and PTEN mutations may result in resistance to EGFR TKI. ${ }^{4}$ In this study, we found PIK3CA and PTEN mutations in seven (4.3\%) and four (2.5\%) patients, respectively. Concurrent EGFR/PIK3CA mutations were detected in three patients. All of them received EGFR TKI ( 2 gefitinib and 1 erlotinib) treatment and showed partial response with different range of PFS (6.6-21.3 months). Concurrent EGFR/PTEN mutations were found in two patients and one received EGFR TKI (afatinib) treatment with partial response (PFS, 8.1 months). However, neither PIK3CA nor PTEN mutation status alone had significant effects on PFS and OS in the EGFR-mutant group. In STK11, we identified mutations in 20 patients (19 F354L and 1 D176G). STK11 encodes the serine/threonine protein kinase and is part of the STK11/AMPK/mammalian target of rapamy- cin signaling pathway. STK11 mutations were commonly found, and inactivation of STK11 is known to promote tumorigenesis and is associated with worse survival outcome. ${ }^{20,28}$ The overall rate of STK11 mutations (12.3\%) was slightly lower than that indicated by The Cancer Genome Atlas (17\%). ${ }^{18}$ This discrepancy can be explained by the origin of the population; STK11 mutations have been reported to be associated with European ancestry. ${ }^{19,21}$ STK11 mutations often coexist with KRAS mutations and have confounding prognostic significance. ${ }^{29,30}$ However, in this study, we found only one concurrent KRAS/STK11 (G12A/ D176G) mutation. This patient received chemotherapy (AP: doxorubicin, cisplatin) and showed partial response with 4.4 months of PFS. A recent study reported that pathogenic STK11 F354L mutations had been recurrently identified in three EGFR TKI non-responders, while these mutations had not been found in EGFR TKI responders. ${ }^{20}$ In our study, seven STK11 F354L mutations were recurrently found in EGRK TKI-treated patients. Among them, six (treated with gefitinib and erlotinib) showed partial response (PFS, 4.1 to 17.8 months) and one (treated with afatinib) showed stable disease (PFS, 19.3 months). This discrepancy may be due to the small sample size of STK11 mutant patients. Thus, more research is required to identify the clinical implications of STK11 mutations.

Our study has a few limitations. Our analysis relied on targeted sequencing to investigate genetic alterations and thus the genes selected in this study may only explain a portion of the total genetic alterations. The NGS platform used in this study (AmpliSeq Cancer Hotspot Panel) detected only SNVs, and thus it was impossible to detect copy number variations $(\mathrm{CNVs})$ and translocations. Furthermore, other novel genetic or epigenetic alterations may have been missed. Most tumor samples were acquired from small biopsy samples, and thus there were not enough tissue available for more comprehensive analysis. Therefore, there is a need for new NGS platforms to simultaneously detect SNVs, CNVs, and translocations, even with small amounts of tissue samples. In addition, functional effects of the detected mutations were not evaluated in vitro.

Our results demonstrate that targeted sequencing using NGS is feasible for mutation profiling of small biopsy samples in NSCLC. We also demonstrated previously unappreciated mutations, enabling further refinements of subclassification for the prediction of therapeutic effects. In conclusion, we suggest that more comprehensive genomic characterizations of NSCLC with small biopsy samples would reveal coexisting alterations that might influence the efficacy of therapy. 


\section{Conflicts of Interest}

No potential conflict of interest relevant to this article was reported.

\section{Acknowledgments}

This research was supported by a grant of the Korea Health Technology R\&D Project through the Korea Health Industry Development Institute (KHIDI), funded by the Ministry of Health \& Welfare, Republic of Korea (grant number: HI16 C1984).

\section{REFERENCES}

1. Jung KW, Won YJ, Oh CM, et al. Prediction of cancer incidence and mortality in Korea, 2016. Cancer Res Treat 2016; 48: 451-7.

2. Chen Z, Fillmore CM, Hammerman PS, Kim CF, Wong KK. Nonsmall-cell lung cancers: a heterogeneous set of diseases. Nat Rev Cancer 2014; 14: 535-46.

3. Richer AL, Friel JM, Carson VM, Inge LJ, Whitsett TG. Genomic profiling toward precision medicine in non-small cell lung cancer: getting beyond EGFR. Pharmgenomics Pers Med 2015; 8: 63-79.

4. Camidge DR, Pao W, Sequist LV. Acquired resistance to TKIs in solid tumours: learning from lung cancer. Nat Rev Clin Oncol 2014; 11: 473-81.

5. Han JY, Kim SH, Lee YS, et al. Comparison of targeted next-generation sequencing with conventional sequencing for predicting the responsiveness to epidermal growth factor receptor-tyrosine kinase inhibitor (EGFR-TKI) therapy in never-smokers with lung adenocarcinoma. Lung Cancer 2014; 85: 161-7.

6. Rathi V, Wright G, Constantin D, et al. Clinical validation of the 50 gene AmpliSeq Cancer Panel V2 for use on a next generation sequencing platform using formalin fixed, paraffin embedded and fine needle aspiration tumour specimens. Pathology 2017; 49: 75-82.

7. Kris MG, Johnson BE, Berry LD, et al. Using multiplexed assays of oncogenic drivers in lung cancers to select targeted drugs. JAMA 2014; 311: 1998-2006.

8. Ballester LY, Luthra R, Kanagal-Shamanna R, Singh RR. Advances in clinical next-generation sequencing: target enrichment and sequencing technologies. Expert Rev Mol Diagn 2016; 16: 357-72.

9. Padmanabhan V, Steinmetz HB, Rizzo EJ, et al. Improving adequacy of small biopsy and fine-needle aspiration specimens for molecular testing by next-generation sequencing in patients with lung cancer: a quality improvement study at Dartmouth-Hitchcock Medical Center. Arch Pathol Lab Med 2017; 141: 402-9.

10. Zheng G, Tsai H, Tseng LH, et al. Test feasibility of next-generation sequencing assays in clinical mutation detection of small biopsy and fine needle aspiration specimens. Am J Clin Pathol 2016; 145: 696-702.

11. Kim ST, Lee J, Hong M, et al. The NEXT-1 (Next generation pErsonalized $\mathrm{tX}$ with mulTi-omics and preclinical model) trial: prospective molecular screening trial of metastatic solid cancer patients, a feasibility analysis. Oncotarget 2015; 6: 33358-68.

12. Kim HJ, Lee KY, Kim YC, et al. Detection and comparison of peptide nucleic acid-mediated real-time polymerase chain reaction clamping and direct gene sequencing for epidermal growth factor receptor mutations in patients with non-small cell lung cancer. Lung Cancer 2012; 75: 321-5.

13. Ku BM, Jung HA, Sun JM, et al. High-throughput profiling identifies clinically actionable mutations in salivary duct carcinoma. J Transl Med 2014; 12: 299.

14. Lee GD, Lee SE, Oh DY, et al. MET exon 14 skipping mutations in lung adenocarcinoma: clinicopathologic implications and prognostic Values. J Thorac Oncol 2017; 12: 1233-46.

15. Karachaliou N, Mayo C, Costa C, et al. KRAS mutations in lung cancer. Clin Lung Cancer 2013; 14: 205-14.

16. Rosell R, Carcereny E, Gervais R, et al. Erlotinib versus standard chemotherapy as first-line treatment for European patients with advanced EGFR mutation-positive non-small-cell lung cancer (EURTAC): a multicentre, open-label, randomised phase 3 trial. Lancet Oncol 2012; 13: 239-46.

17. Kuan FC, Kuo LT, Chen MC, et al. Overall survival benefits of firstline EGFR tyrosine kinase inhibitors in EGFR-mutated non-smallcell lung cancers: a systematic review and meta-analysis. Br J Cancer 2015; 113: 1519-28.

18. Cancer Genome Atlas Research Network. Comprehensive molecular profiling of lung adenocarcinoma. Nature 2014; 511: 543-50.

19. Dearden S, Stevens J, Wu YL, Blowers D. Mutation incidence and coincidence in non small-cell lung cancer: meta-analyses by ethnicity and histology (mutMap). Ann Oncol 2013; 24: 2371-6.

20. Lim SM, Kim HR, Cho EK, et al. Targeted sequencing identifies genetic alterations that confer primary resistance to EGFR tyrosine kinase inhibitor (Korean Lung Cancer Consortium). Oncotarget 2016; 7: 36311-20.

21. Mäki-Nevala S, Sarhadi VK, Rönty M, et al. Hot spot mutations in Finnish non-small cell lung cancers. Lung Cancer 2016; 99: 102-10.

22. Murakami I, Hiyama K, Ishioka S, Yamakido M, Kasagi F, Yokosaki Y. p53 gene mutations are associated with shortened survival in patients with advanced non-small cell lung cancer: an analysis of medically managed patients. Clin Cancer Res 2000; 6: 526-30.

23. Lim EH, Zhang SL, Li JL, et al. Using whole genome amplification (WGA) of low-volume biopsies to assess the prognostic role of 
EGFR, KRAS, p53, and CMET mutations in advanced-stage nonsmall cell lung cancer (NSCLC). J Thorac Oncol 2009; 4: 12-21.

24. Glubb DM, Cerri E, Giese A, et al. Novel functional germline variants in the VEGF receptor 2 gene and their effect on gene expression and microvessel density in lung cancer. Clin Cancer Res 2011; 17: 5257-67.

25. Silva IP, Salhi A, Giles KM, et al. Identification of a novel pathogenic germline KDR variant in melanoma. Clin Cancer Res 2016; 22: 2377-85.

26. Roberts PJ, Stinchcombe TE. KRAS mutation: should we test for it, and does it matter? J Clin Oncol 2013; 31: 1112-21.

27. Kim HR, Cho BC, Shim HS, et al. Prediction for response duration to epidermal growth factor receptor-tyrosine kinase inhibitors in EGFR mutated never smoker lung adenocarcinoma. Lung Cancer 2014; 83: 374-82.

28. Pécuchet N, Laurent-Puig P, Mansuet-Lupo A, et al. Different prognostic impact of STK11 mutations in non-squamous non-small-cell lung cancer. Oncotarget 2017; 8: 23831-40.

29. Gleeson FC, Kipp BR, Levy MJ, et al. Somatic STK11 and concomitant STK11/KRAS mutational frequency in stage IV lung adenocarcinoma adrenal metastases. J Thorac Oncol 2015; 10: 531-4.

30. Imielinski M, Berger AH, Hammerman PS, et al. Mapping the hallmarks of lung adenocarcinoma with massively parallel sequencing. Cell 2012; 150: 1107-20. 